\title{
\#Diversity: Conversations on Twitter about Women and Black Men in Medicine
}

\author{
Ranit Mishori, MD, MHS, FAAFP, Lisa Singh, PhD, \\ Kenneth W. Lin, MD, MPH, FAAFP, and Yifang Wei, PhD
}

Background: Discussions about racism, ethnicity, sexism, discrimination, and diversity have increased within medicine, and their impact on the physician workforce, advancement, hiring, wage inequities, mistreatment, and scholarly output, to name a few. Most medical organizations have created policies and initiatives on diversity and inclusion, focusing on supporting underrepresented minorities. Similar discussions are taking place online, including on Twitter, via specific hashtags, such as \#BlackMenInMedicine, \#ILookLikeASurgeon. News reports suggested some of these hashtags were "trending." We set out to assess selected hashtags and analyze their spread, as well as whether or how health professional organizations publicized or amplified this emerging discourse on Twitter.

Methodology: We computed tweet volume, retweet volume impressions, and spread for selected hashtags and for health-profession organizations.

Results: The overall volume was average or below average when compared with all active Twitter users; however, the retweet percentage was $60 \%$, suggesting high levels of engagement. There was modest spread of most of the messages containing the hashtags, with the exception of \#ilooklikeasurgeon tweets, due to its relationship to the cover of a major nonmedical magazine. Spread for some hashtags, despite very low initial retweets, was increased due to retweeting by accounts with high volume of followers. Medical societies' contributions to dissemination were very minor.

Conclusion: Strengthening, deepening and, ultimately, expanding the conversation on diversity and inclusion in medicine on Twitter requires an intentional and strategic use of hashtags, photographs and links; Engaging "influencers" such as mainstream media and medical organizations is also critical to more widespread dissemination. (J Am Board Fam Med 2019;32:28-36.)

Keywords: Medical Societies, Physicians, Racism, Sexism, Social Media, Twitter

Historically, women and Black men have been underrepresented in medicine and the targets of discrimination in hiring, wages, harassment, and professional advancement opportunities. ${ }^{1,2}$ Not only is a lack of diversity among physician leaders problematic for the medical profession, it may have

This article was externally peer reviewed.

Submitted 17 June 2018; revised 3 September 2018; accepted 6 October 2018.

From the Department of Family Medicine (RM, KWL), Department of Computer Science (LS, YW), Georgetown University School of Medicine, Washington, DC.

Funding: none.

Conflict of interest: none declared.

Corresponding author: Ranit Mishori, MD, MHS, FAAFP, Georgetown University School of Medicine, 3900 Reservoir Rd. NW, Washington, DC 20007 (E-mail: mishorir@ georgetown.edu). adverse impacts on the health and wellbeing of patients. ${ }^{3,4}$

To raise awareness of this problem and reach stakeholders within the worlds of academia, medicine and health care in general, medical organizations have created policies and initiatives on diversity and inclusion that focus on improving opportunities for female and minority physicians. ${ }^{5-12}$

Since its launch in 2006, Twitter has allowed everyone, including physicians, to exchange ideas with colleagues, the public, and other stakeholders $^{13-15}$ and create virtual communities. ${ }^{16,17}$ Twitter users can post messages ("tweets") of up to 280 characters that other users can share ("retweet") to their followers, greatly magnifying the messages' spread. As regular users (RM and KL), we noticed a growing Twitter discourse on racism, discrimination, and diversity in medicine, including online 


\begin{tabular}{|c|c|}
\hline Term & Description \\
\hline Twitter user & A user who has a unique account \\
\hline Twitter handle/username & $\begin{array}{l}\text { The name and/or identity of a user on Twitter (e.g., @STFM_FM), applies to individuals } \\
\text { as well as organizations and businesses }\end{array}$ \\
\hline Tweet & A message limited to 280 characters posted by a Twitter user \\
\hline Retweet (RT) & A tweet that is forwarded by a Twitter user who did not author the original tweet \\
\hline Follower & A user who receives all of the tweets of the Twitter user account holder \\
\hline Hashtag & $\begin{array}{l}\text { A topic or event that a user wants to send a tweet about. To easily identify it, the \# } \\
\text { symbol precedes the topic word/phrase (e.g., \#womenInMedicine) }\end{array}$ \\
\hline Tweet initial impressions & The number of users who see the tweet when it is initially posted \\
\hline Tweet spread & The number of people who see the tweet initially and after it is propagated (or retweeted) \\
\hline Trending & $\begin{array}{l}\text { Topics that-according to specific algorithms tailored to individual users, are popular at a } \\
\text { given time. Trends are decided based on sharp spikes on a topic compared to the norm. }\end{array}$ \\
\hline Viral & A tweet that spreads quickly to a large number of users \\
\hline Connectedness & $\begin{array}{l}\text { A measure and spatial representation of the connectivity of users and followers and the } \\
\text { relationship between them. }\end{array}$ \\
\hline
\end{tabular}

campaigns to promote gender and racial equality and equity via specific hashtags (see Table 1 for definitions). News reports suggest that some of these hashtags were "trending"18-21_ or spiking in frequency and number compared with the norm. ${ }^{22}$ We wanted to assess how such conversations circulate on Twitter by analyzing some common metrics, such as the volume, dissemination, and content of hashtags on women and Black men in medicine. We also wanted to determine whether or how mainstream health professional organizations representing the majority of US-based physicians or specific subgroups of physicians, engage in or help expand this emerging discourse on Twitter.

\section{Methodology}

This project is based on publicly available data. Because no private data was collected, maintained, or inferred, this project and thus did not meet criteria for Institutional Review Board (IRB) review. We selected the specific hashtags based on anecdotal popularity (see Table 2), and confirmed their inclusion on the Health Care Hashtag Project, which curates common, emerging, and popular hashtags in health care. This hashtag project is run by Symplur, a social media analytics company that specializes in health care (https://www.symplur.com/healthcare-hashtags/).

\section{Data Collection}

We analyzed the appearance of these hashtags in tweets between January 1, 2017 and December 31, 2017. We collected tweets containing one or more of the hashtags using the Twitter Application Programming Interface (https://developer.twitter.com/ en/docs) - the standard way Twitter has developed for researchers to access data. We also collected basic user profiles of the individuals who posted the tweets, including their handles and number of followers. Finally, we also collected the basic user profile information of medical organizations and the tweets that they posted in 2017. These organizations were selected based on their prominence in the field, and representation of a diverse membership, that includes women and black men.

\section{Variables/Metrics}

We constructed the following variables for each selected hashtag: volume of tweets per month (tweet volume), volume of retweets per month (retweet volume), initial impressions per month (initial impressions), impressions resulting from initial tweet and retweets per month (spread), number of distinct contributors using the hashtag in one or more tweets (contributors), and counts of the most frequent words used for each hashtag (frequent words). We also computed aggregates of these variables across all the hashtags in our dataset. The number of initial impressions for a single tweet represents the number of individuals who see the tweet when it is posted by a user $(U)$. While many different methods for computing impressions have been proposed, Twitter defines an impression as the number of times a user is shown a tweet in their timeline or during search results. ${ }^{23}$ Because we do not have access to Twitter search results, we use the 


\begin{tabular}{|c|c|c|}
\hline Hashtag & Description and Curated in Healthcare Hashtags & $\begin{array}{c}\text { Year Hashtag Started } \\
\text { Being Used (Volume } \\
\text { That Year) }\end{array}$ \\
\hline \#ilooklikeasurgeon & $\begin{array}{l}\text { Created by women surgeons and related to equity and equality for } \\
\text { women in the field of surgery. (Curated in Symplur). }\end{array}$ & $2013(<5)$ \\
\hline \#WomenInMedicine & $\begin{array}{l}\text { From a weekly tweetchat discussing issues that women face in } \\
\text { medicine. "The goals . . to provide a safe space for the } \\
\text { respectful discussion of issues of gender in medicine . . . an } \\
\text { environment that will hopefully begin to shift the culture of } \\
\text { medicine forward." (Curated in Symplur) }\end{array}$ & $2010(<5)$ \\
\hline \#SheLeads2017 & $\begin{array}{l}\text { Conference hashtag of a Harvard Medical School CME/CNE } \\
\text { course for physicians, nurses and other healthcare leaders that } \\
\text { focuses on professional development and networking, diversity } \\
\text { and inclusion. (Curated in Symplur). }\end{array}$ & $2016(<5)$ \\
\hline \#seeitbeit & $\begin{array}{l}\text { About women and minority empowerment through role models of } \\
\text { the same gender/race/ethnicity. (Not appearing on Symplur). }\end{array}$ & $2010(<5)$ \\
\hline \#BlackMenInMedicine & $\begin{array}{l}\text { Even though it was first used in } 2013 \text {, it did not gain traction } \\
\text { until a presentation on the number of African American men in } \\
\text { medical school in } 2017 \text {. Addresses racial diversity for black } \\
\text { males. (Curated in Symplur). }\end{array}$ & $2013(<5)$ \\
\hline \#QuoteHer & $\begin{array}{l}\text { Related to leadership for women in medicine and science and } \\
\text { women as experts. (Curated in Symplur.) }\end{array}$ & $2009(<50)$ \\
\hline \#diversityinmedicine & $\begin{array}{l}\text { General catchphrase about diversity in medicine. Does not appear } \\
\text { in Symplur. }\end{array}$ & $2012(<5)$ \\
\hline
\end{tabular}

definition of impressions proposed by the health care analytic website, Symplur since that definition has been used in different health-related studies. $^{24,25}$ Symplur defines impressions as an estimate of the number of times a tweet may be viewed and calculates it by multiplying the number of tweets posted that use specific hashtags by user $U$ by the number of followers (f) for that user, and then adds these numbers across all user during the analysis time period. More formally, we compute the initial impressions for a hashtag, I(h), as follows:

$$
I(b)=\sum_{j=1}^{n}\left|U_{j}(f)\right|
$$

where $n$ is the number of tweets and $\left|U_{j}(f)\right|$ is the number of followers of user $U_{j}$.

This particular definition of impressions does not include retweet information explicitly. Therefore, we extend the notion of impressions to explicitly incorporate impressions related to retweets. The spread $(S)$ of a hashtag is computed by combining the initial impressions to the impressions resulting from retweets. More formally, we compute the spread for a hashtag, $\mathrm{S}(\mathrm{h})$, as follows:

$$
S(b)=\sum_{j=1}^{n}\left(\left|U_{j}(f)\right|+\sum_{k=1}^{r_{j}}\left|U_{k}^{\prime}(f)\right|\right)
$$

where $\mathrm{h}$ is a specific hashtag, $\mathrm{n}$ is the number of tweets, $r_{j}$ is the number of times the $j^{\text {th }}$ tweet was retweeted, $U^{\prime}$ is a follower of $U$ who retweeted $U$ 's tweet and $\left|U^{\prime}{ }_{k}(f)\right|$ is the number of followers for $\mathrm{U}^{\prime}$.

We approximated $\left|U^{\prime}{ }_{k}(f)\right|$ using the median number of followers for the users who post a tweet containing 1 of our medical diversity hashtags. We use the median because the distribution is skewed and the mean may give a figure that is large. We used the same variables for the professional associations, and included the proportion of all tweets posted by the professional association containing a selected hashtag.

\section{Results}

\section{Spread and Dissemination}

Table 3 shows the overall volume of tweets and retweets that used the selected hashtags in 2017, which is fairly average when compared with all active Twitter users (the average active user sends approximately 550 tweets per year), ${ }^{26}$ but modest when considering active hashtags (the \#metoo hashtag has been included in over 18 million tweets since October 2017). There was modest spread associated with these messages, with a ratio of retweets to tweets ranging from 1.5 to 6 . The notable exceptions were the \#ilooklikeasurgeon tweets involving a cover of The New Yorker (from 
Table 3. Volume of Tweets and Retweets Using Diversity Hashtags from January 1 to December 31, 2017

\begin{tabular}{lrrrrr}
\hline Hashtag & Total & & \multicolumn{2}{c}{ Total } & \multicolumn{2}{c}{$\begin{array}{c}\text { Initial } \\
\text { Impressions }\end{array}$} & Spread \\
\hline \#weets & Contributors & 2181 & 60,643 & $2,707,841$ & $4,750,993$ \\
\#WomenInMedicine & 11,582 & 1087 & 14,650 & $1,952,259$ & $3,138,873$ \\
\#SheLeads2017 & 5094 & 301 & 7607 & 313,693 & 487,490 \\
\#seeitbeit & 2719 & 176 & 1519 & $12,861,874$ & $13,562,133$ \\
\#BlackMenInMedicine & 1079 & 151 & 4432 & $8,629,263$ & $12,136,090$ \\
\#QuoteHer & 737 & 146 & 377 & $163,620,576$ & $191,576,999$ \\
\#diversityinmedicine & 649 & 142 & & $13,275,032$ & $20,028,682$ \\
\hline
\end{tabular}

April 2017) that featured the masked faces of 4 female surgeons looking down at an operating field. ${ }^{27}$ This cover photograph appeared on the timelines of millions of users.

The overall retweet impressions of these hashtags were between just under 500 thousand and 5 million. This gives us an estimate of how many additional accounts were seeing the tweet. These figures also show that the median number of followers for the users posting tweets with the hashtags was 461 and the average was 8163 . In comparison, in 2013, the median number of followers for users posting in the previous 30 days was $61,{ }^{28}$ and using Twitter statistics released in 2017, this mean is higher than that of the normal user on Twitter, which is $707 .^{26}$ The 22,019 tweets sent containing one of the medical diversity hashtags in 2017 were sent by 5683 different Twitter users. Sixty-eight of these users used one or more of these hashtags in more than 50 tweets. Of the 22,019 tweets, over $60 \%$ were retweeted. Figure 1 shows the various hashtags and variations in volume and activity by month for 2017.

\section{Connectedness}

Looking at "connectedness"-a measure and spatial representation of the connectivity between users and followers and the bidirectional relationship between them-we analyzed the Twitter activity of several health professional groups to see if/how they were using the hashtags in their tweets. Collectively, these organizations tweeted between 995 and 2197 times each month in 2017. Overall, tweets using the selected hashtags made up a minuscule proportion of their overall tweets (Table 4), with the exception of tweets from the Association of Women Surgeons, which used one of the hashtags in $11 \%$ of its tweets.
Figure 2 further illustrates how little overlap occurred between professional societies and users of the diversity hashtags. Lines are drawn between the professional societies and the diversity hashtags if the professional society posted tweets that contain the specified hashtag. @WomenSurgeons is the only society that has used most of the hashtags in tweets they have posted.

\section{Discussion}

Physicians use Twitter to share scholarship, comment on research, engage with colleagues and the public, create virtual communities, and build new social networks. ${ }^{28-31}$ Increasingly, many are using Twitter to reflect on personal experiences, advocate for change, and engage in discussions surrounding racism, discrimination, and sexism in medicine. ${ }^{16}$ Our study is the first to characterize Twitter discourse on women and Black men in medicine via commonly used hashtags.

Overall, the use of these hashtags has been limited, but it has been growing. One notable exception is \#ILookLikeASurgeon. Interestingly, that campaign started in 2015,,$^{31,32}$ but in April 2017 it was adopted by Twitter users to go along with the New Yorker cover and the ensuing photographs sent from around the world of women surgeons. ${ }^{33}$ As a means of comparison, the top tweets on Twitter are retweeted over a half million times. In contrast, the top tweets in our evaluation were retweeted a mere 500 times. At the same time, the "retweet percentage" of tweets with these hashtags was $60 \%$. This represents a very high level of engagement, since overall, only $1 \%$ of tweets on Twitter are retweeted. ${ }^{34}$

We also see examples of hashtags with a relatively small number of tweets initially (\#QuoteHer and \#diversityinmedicine), ending up with a large spread. This is due to who was retweeting or using 
Figure 1. Hashtags and their volume by month for 2017.
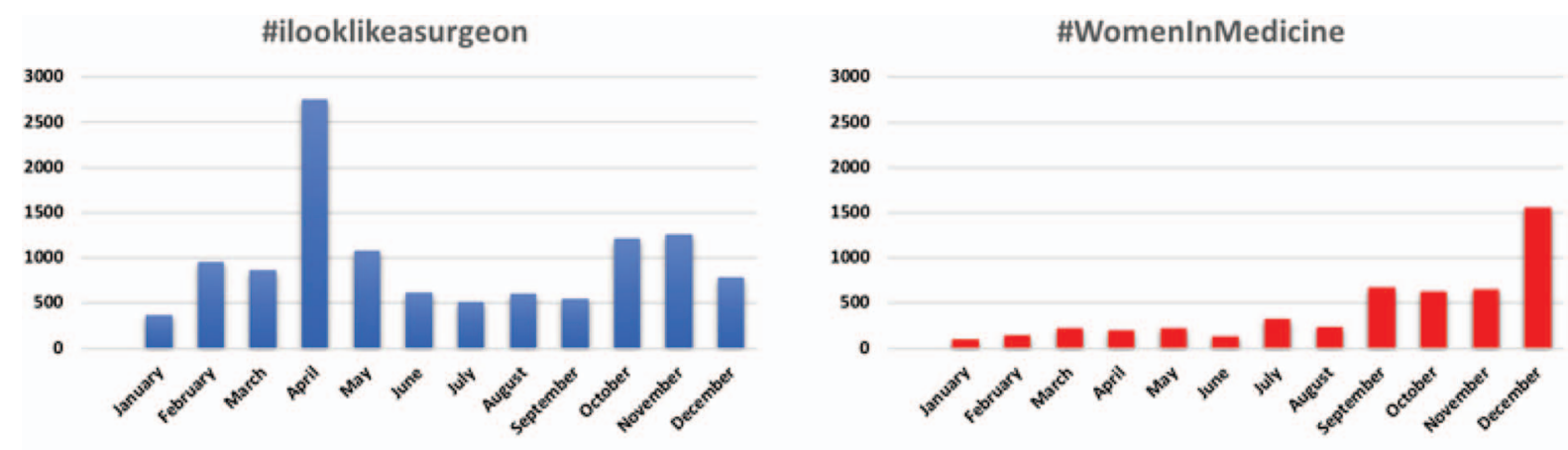

\#SheLeads2017

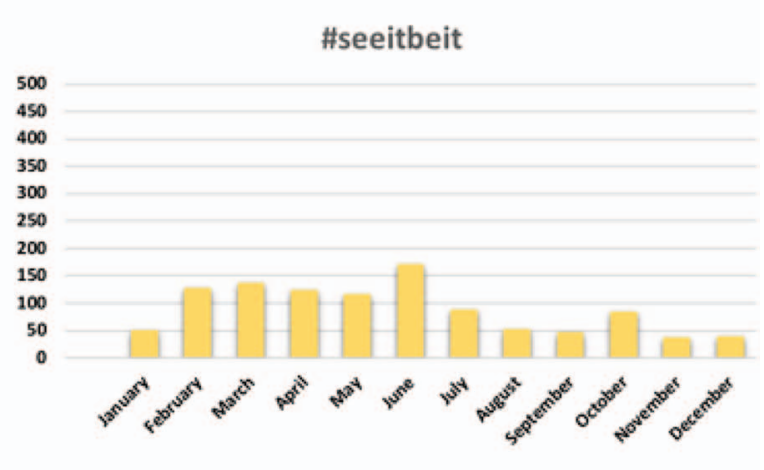

\#BlackMenInMedicine

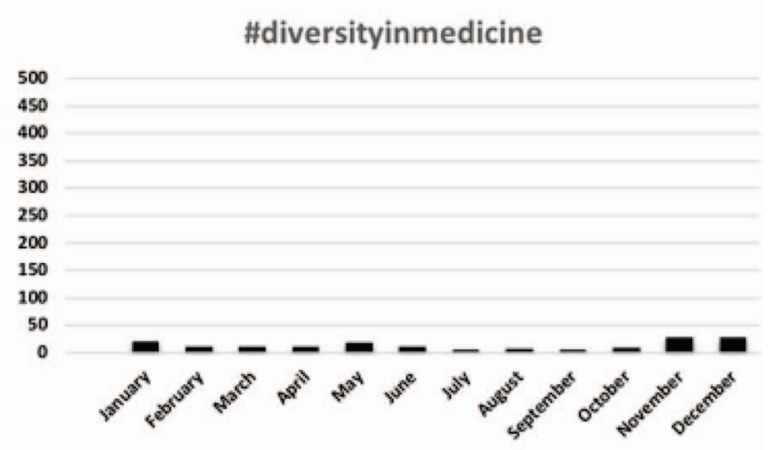

\#QuoteHer
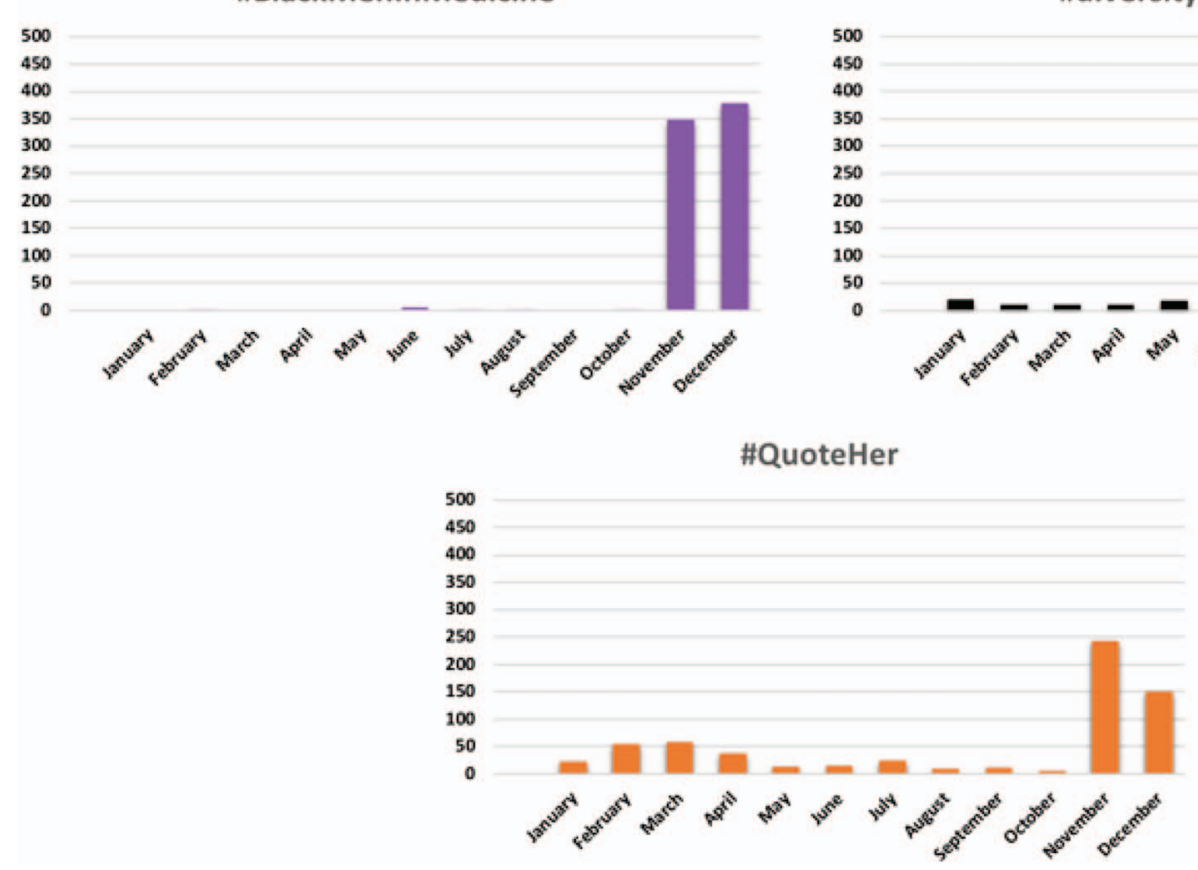

the hashtag. \#QuoteHer was used by 5 handles that had over 100 thousand followers each (@harvardmed, @womensmediacntr, @Medscape, @CJR, @DrLindaMD). For \#diversityimedicine, we did not see many users with 100 thousand followers, but they had a few influential users, including @ picardonhealth (a well known health journalist with $>70$ thousand followers) and @BlackWomenMDs (with about 10 thousand followers) tweeted repeatedly using the hashtag.

While this is a start, these conversations have not reached their potential, although it is unlikely they would become as large as \#metoo or \#blacklivesmatter or others that have gone truly "viral." 
Table 4. Medical Groups' Use of Diversity Hashtags between January to December 2017

\begin{tabular}{|c|c|c|c|c|}
\hline Handle & $\begin{array}{c}\text { Diversity Tweet } \\
\text { Volume }\end{array}$ & $\begin{array}{l}\text { Overall } \\
\text { Tweets }\end{array}$ & $\begin{array}{l}\text { Overall } \\
\text { Retweets }\end{array}$ & $\begin{array}{l}\text { Diversity Tweet } \\
\text { Significance }\end{array}$ \\
\hline$@$ aafp & 0 & 4314 & 10731 & 0 \\
\hline @ACPinternists & 1 & 1100 & 3299 & $<1 \%$ \\
\hline @AmCollSurgeons & 0 & 3050 & 11391 & 0 \\
\hline @AmerAcadPeds & 0 & 1819 & 80404 & 0 \\
\hline @AmerMedicalAssn & 4 & 2665 & 60303 & $<1 \%$ \\
\hline @AMSANational & 3 & 721 & 720 & $<1 \%$ \\
\hline @AMWADoctors & 15 & 272 & 183 & $6 \%$ \\
\hline @NationalMedAssn & 0 & 485 & 471 & 0 \\
\hline @SocietyGIM & 0 & 490 & 544 & 0 \\
\hline @societyofBAS & 9 & 248 & 537 & $4 \%$ \\
\hline @STFM_FM & 0 & 906 & 1179 & 0 \\
\hline @WomenSurgeons & 209 & 1862 & 8399 & $11 \%$ \\
\hline
\end{tabular}

However, for such hashtags (and associated tweets, themes and ideas) to go viral just within our medical community, more people and professional societies need to use them, we need to target "influencers" or those with a large following, and we need to be persistent in tweeting and retweeting frequently and repeatedly.
Events "in real life" (IRL) drive content on Twitter. Spikes in the \#ILookLikeASurgeon were linked to the publication of the New Yorker cover cartoon in April 2017; The \#BlackMenInMedicine had its spike in November 2017, coinciding with a campaign on racial diversity in medicine promoted by an organization called "Tour for Diversity in

Figure 2. Connectedness and amplification: medical societies' and organizations' use of selected hashtags.
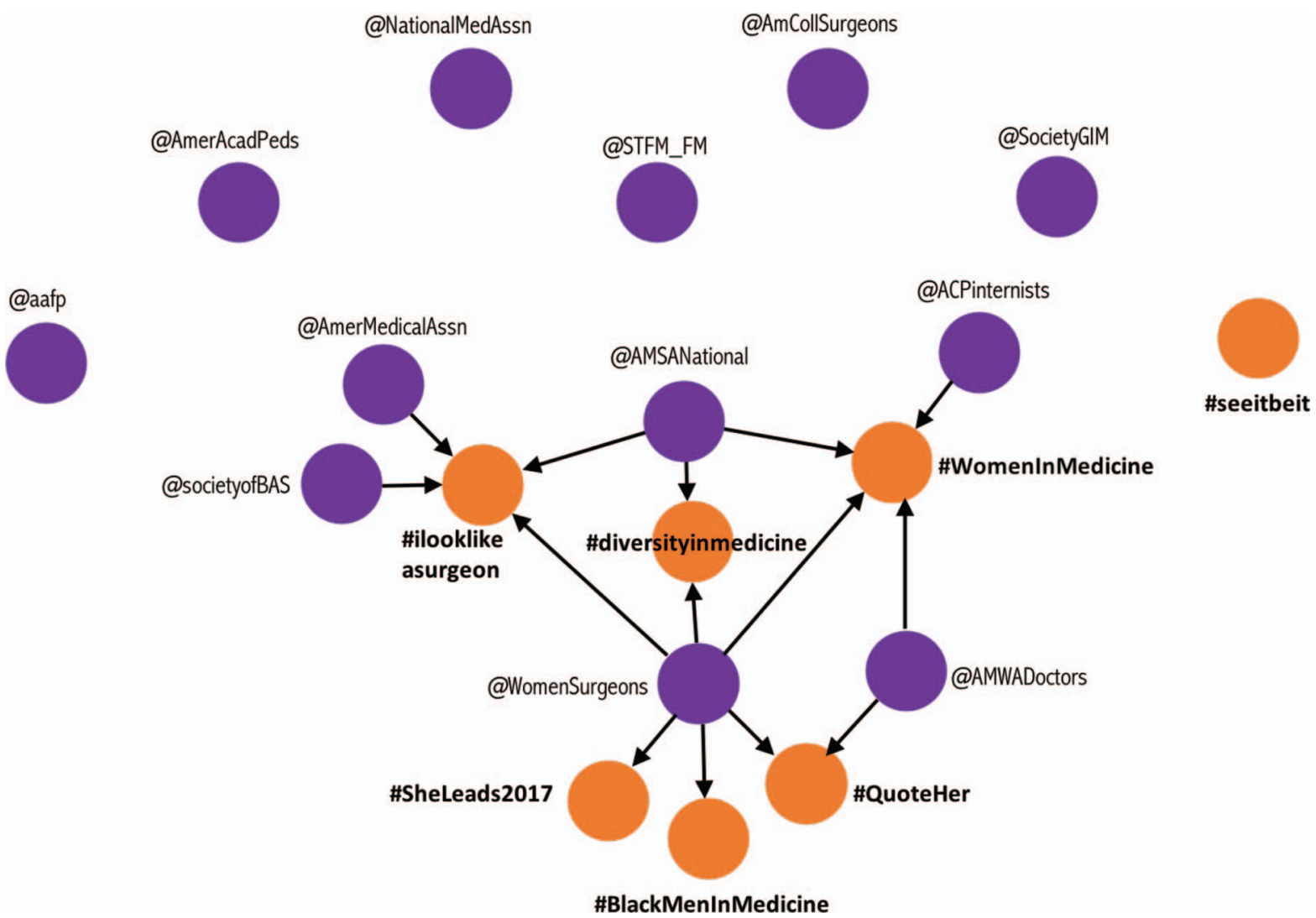
Medicine." 35 The \#SheLeads hashtag showed increased popularity just before and during a conference $^{36}$; the \#womenInMedicine hashtags, used during weekly tweetchats, also peaked in April, perhaps used along with the \#ILookLikeASurgeon hashtag.

The temporal variations illustrate how difficult it is to sustain the momentum of such "campaigns" over a long period of time, and attest to the fleeting nature of "organized hashtags," especially since any user can create their own hashtag and enough people need to know about the hashtag and copy it into their tweets to matter. The smallest variations in hashtag composition (eg, \#pediatrics, \#pediatric, \#pediatrician), sometime related to typing errors, mean that tagged tweets will not be curated as part of the same "conversation," making it more difficult for researchers to track the real spread and distribution.

One way to sustain a conversation is to have organizations, known entities or health care leaders start using a hashtag to tag specific issues. Very few health professional groups used any of the selected hashtags, and those that did, did so rarely. This omission represents a missed opportunity to not only amplify important on-line conversations, but to lead them. It may send the impression that the diversity and inclusion are not priorities for the selected organizations. For such hashtags to be essentially ignored by Twitter influencers from within the medical community will essentially guarantee that important content will remain confined to small silos and closed communities. Health profession organizations have the opportunity to amplify messages related to racism, sexism, discrimination and diversity in an intersectional and cross-disciplinary manner. Most of them have thousands, if not hundreds of thousands of followers (eg, the American Medical Association has over 650 thousand followers).

Our study has several limitations. First and foremost, this evaluation represents data from a limited time period. Content and context continuously evolve on social media, so while this investigation looked at an entire year, it may not be representative of trends in the present and future. Second, our focus on hashtags means that we only captured a limited number of tweets - and a narrower discourse - and may have missed important discussions in tweets without the selected hashtags. In addition, social media conversations about diversity, racism and discrimination in medicine occur "outside" of the selected hashtags by individuals and organizations that do not use them (purposely or simply because they are not aware of them) or use them incorrectly.

Moreover, sometimes campaigns use multiple hashtags (\#BMIM is sometimes used to denote \#BlackMenInMedicine), further limiting a comprehensive assessment. What is more, our selection of hashtags represents our own notion of what is important and is limited to our personal exposures in our personal Twitter feeds. As such, we may have omitted other important hashtags, affecting how representative our selections are of all hashtags related to diversity in medicine. Further, we did not include all health-profession organizations, universities, medical schools or other societies with a stake in this conversation. Finally, we also did not conduct subanalyses of the effects of other factors affecting the dissemination and spread such as the inclusion of photographs, links or tweets' time of day (all which have been linked to dissemination potential $^{37}$ ).

By and large, however, the study of the impact of social media discourse in health and medicine- on patient outcomes, professional and patient behavior, dissemination of research-is still in its infancy. ${ }^{38-41}$

\section{Conclusion}

Discussions surrounding racism, sexism, and discrimination in medicine are gaining momentum and reaching wider audiences within our profession. The conversation can be broadened to reach across specialties, geographical locations, and even beyond medicine through the use of social media, but we must be more intentional in our use of Twitter, and of hashtags, for this purpose. Recent data has shown that the use of hashtags increases engagement, as does the use of images and links. ${ }^{37}$

For researchers being intentional may mean creating more uniform means of quantifying and assessing dissemination via hashtags. ${ }^{37}$ On the individual user level it may mean using photographs, linking to relevant web sites, noticing emerging diversity-related hashtags, using them purposefully, tagging multiple organizations and Twitter "influencers." It may also mean contributing new hashtags to the Health Care Hashtag Project, ${ }^{42}$ a "social project" soliciting input from users to curate "trending" data and Internet discourse via hashtags. 
Individuals and organizations have the potential to amplify and broaden awareness of obstacles faced by women and Black men in medicine. It may be in the best interest of organizations to become aware of what their members and others in the field are tweeting, what hashtags are being used and how the discourse on inclusion and diversity is shifting. We would like to see health care organizations, however, also take an active role in strengthening, deepening and, ultimately, expanding the conversation not only on Twitter, but more importantly IRL-in real life. Diversity and inclusion in medicine must go viral, on and off the Internet.

We would like to acknowledge the Massive Data Institute (MDI) at Georgetown University for supporting this research.

To see this article online, please go to: bttp://jabfm.org/content/ 32/1/28.full.

\section{References}

1. Rotenstein LS, Jena AB. Lost Taussigs-the consequences of gender discrimination in medicine. N Engl J Med 2018;378:2255-7.

2. Laurencin CT, Murray M. An American crisis: The lack of Black men in medicine. J Racial Ethn Health Disparities 2017;4:317-21.

3. Alsan M, Garrick O, Graziani GC. Does diversity matter for health? Experimental evidence from Oakland. Working Paper 24787. National Bureau of Economic Research; 2018.

4. Cohen JJ, Gabriel BA, Terrell C. The case for diversity in the health care workforce. Health Affairs 2002;21:90-102.

5. American Medical Association. Minority affairs section. Available from: https://www.ama-assn.org/ about/minority-affairs-section-mas. Accessed February 27, 2018.

6. Association of American Medical Colleges (AAMC). Diversity and inclusion initiatives. Available from: https://www.aamc.org/initiatives/diversity/. Accessed February 27, 2018.

7. American Medical Student Association. AMSA (blog). Diversity. Available from: https://www. amsa.org/about/mission-aspirations/diversity/. Accessed February 27, 2018.

8. American Medical Women's Association (blog). Diversity and Inclusion. Available from: https://www. amwa-doc.org/our-work/initiatives/diversity-andinclusion/. Accessed February 27, 2018.

9. American Academy of Family Physicians. Center for Diversity and Health Equity. Available from: https:// www.aafp.org/patient-care/social-determinants-ofhealth/cdhe.html. Accessed February 27, 2018.

10. AAP Diversity and Inclusion Statement. American Academy of Pediatrics. AAP News, February 26,
2018. Available from: http://www.aappublications. org/news/2017/10/30/DiversityStatement103017.

11. Importance of Social Determinants of Health and Cultural Awareness in the Delivery of Reproductive Health Care-American College of Obstetrics and Gynecology (ACOG). Available from: https:// www.acog.org/Clinical-Guidance-and-Publications/ Committee-Opinions/Committee-on-Health-Carefor-Underserved-Women/Importance-of-SocialDeterminants-of-Health-and-Cultural-Awarenessin-the-Delivery-of-Reproductive. Accessed February 27, 2018.

12. American College of Surgeons. Committee on Diversity Issues. Available from: https://www.facs.org/ about-acs/governance/acs-committees/committeeon-diversity-issues. Accessed February 27, 2018.

13. Twitter is trending in academic medicine. Available from: https://news.aamc.org/medical-education/ article/twitter-trending-academic-medicine/. Accessed February 8, 2018.

14. Should medical conferences ease social media, photo restrictions? Available from: https://www.medscape. com/viewarticle/881675. Accessed February 27, 2018.

15. Number of healthcare professionals on Twitter tops 75,000. Text. PMLive, April 22, 2014. Available from: http://www.pmlive.com/blogs/digital_intelligence/ archive/2014/april/number_of_healthcare_professionals_ on_twitter_tops_75,000_561975.

16. Shillcutt SK, Silver JK. Social Media and advancement of women physicians. N Engl J Med 2018;378:2342-5.

17. Rolls K, Jackson HM, Elliott D. How health care professionals use social media to create virtual communities: An integrative review. J Med Internet Res 2016;(6):e166.

18. Tour for Diversity (blog). \#BMIM Black males in medicine: The introduction. November 27, 2017. Available from: http://tour4diversity.org/bmimblack-males-medicine-introduction/.

19. Columbia Journalism Review. Campaign \#QuoteHer aims to get women's voices in healthcare reporting. Available from: https://www.cjr.org/analysis/quoteherreporting.php?utm_source $=$ twitter\&utm_medium $=$ social\&utm_campaign $=$ hms-twitter-general. Accessed December 11, 2017.

20. Toronto Star. Can hashtags improve diversity in medicine? Available from: https://www.thestar.com/ opinion/contributors/2017/12/01/can-hashtagsimprove-diversity-in-medicine.html. Accessed December 11, 2017.

21. Townsend T. Social media campaign designed to get more black men interested in medicine. WBNS-10TV Columbus, Ohio. Columbus News, Weather \& Sports. December 11, 2017. Available from: https:// www.10tv.com/article/social-media-campaigndesigned-get-more-black-men-interested-medicine.

22. Twitter Inc. To trend or not to trend. Twitter, December 8, 2010. Available from: https://blog. 
twitter.com/official/en_us/a/2010/to-trend-ornot-to-trend.html. Accessed August 21, 2018.

23. Twitter Inc. About your activity dashboard. Twitter. Available from: https://help.twitter.com/en/managingyour-account/using-the-tweet-activity-dashboard. Accessed August 21, 2018.

24. Ferguson C, Inglis SC, Newton PJ, Cripps PJS, Macdonald PS, Davidson PM, Social media: A tool to spread information: A case study analysis of Twitter conversation at the Cardiac Society of Australia \& New Zealand 61st Annual Scientific Meeting 2013. Collegian 2014;21:89-93.

25. Nason GJ, O'Kelly F, Bouchier-Hayes D, Quinlan DM, Manecksha RP. Twitter expands the reach and engagement of a national scientific meeting: The Irish Society of Urology. Ir J Med Sci 2015;184:685689.

26. Brandwatch (blog). 44 incredible and interesting twitter statistics. December 17, 2017. Available from: https://www.brandwatch.com/blog/44-twitter-stats/.

27. Medscape Medical News. \#ILookLikeASurgeon gets new boost from New Yorker cover. Available from: https://www.medscape.com/viewarticle/878843. Accessed February 26, 2018.

28. Markham MJ, Gentile D, Graham DL. Social media for networking, professional development, and patient engagement. Am Soc Clin Oncol Educ Book 2017;37:782-7.

29. Pershad Y, Hangge PT, Albadawi H, Oklu R. Social medicine: Twitter in healthcare. J Clin Med 2018;7: 121.

30. Choo EK, Ranney ML, Chan TM, et al. Twitter as a tool for communication and knowledge exchange in academic medicine: a guide for skeptics and novices. Med Teach 2015;37:411-6.

31. The Bulletin (blog). \#ILookLikeASurgeon goes viral: How it happened. November 1, 2015. Available from: http://bulletin.facs.org/2015/11/ilooklikea surgeon-goes-viral-how-it-happened/.

32. Symplur. \#ILookLikeASurgeon-Healthcare social media analytics and transcripts. August 7, 2015. Available from: https://www.symplur.com/healthcare-hashtags/ilooklikeasurgeon/.
33. Mouly F, Bormes, G. The New Yorker cover that's being replicated by women surgeons across the world. The New Yorker. Available from: https:// www.newyorker.com/culture/culture-desk/the-newyorker-cover-thats-being-replicated-by-womensurgeons-across-the-world. Accessed August 27, 2018.

34. Stone Temple. Twitter engagement unmasked: A study of more than $4 \mathrm{~m}$ tweets. December 11, 2014. Available from: https://www.stonetemple.com/ twitter-engagement-umasked/.

35. Tour for Diversity (blog). \#BMIM Black males in medicine: The introduction. November 27, 2017. Available from: http://tour4diversity.org/bmimblack-males-medicine-introduction/.

36. She Leads Healthcare. A continuing medical education course for women in healthcare. Available from: http://sheleadshealthcare.com/. Accessed March 3, 2018.

37. Ma Z, Sun A, Cong G. On predicting the popularity of newly emerging hashtags in twitter. J Am Soc Inform Sci Tech 2013;64:1399-410.

38. Pinho-Costa L, Yakubu K, Hoedebecke K, et al. Healthcare hashtag index development: Identifying global impact in social media. J Biom Informatics 2016;63;390-99.

39. Pulido CM, Redondo-Sama G, Sordé-Martí T, Flecha R. Social impact in social media: A new method to evaluate the social impact of research. PloS One 2018;13(8):e0203117.

40. Freeman B, Potente S, Rock V, McIver J. Social media campaigns that make a difference: What can public health learn from the corporate sector and other social change marketers? Public Health Res Pract 2015;25:e2521517.

41. Wekerle C, Vakili N, Stewart SH, Black T. The utility of twitter as a tool for increasing reach of research on sexual violence. Child Abuse Negl 2018; 85:220-8.

42. Lee TM. Healthcare hashtags-A social project. Symplur. October 26, 2010. Available from: https:// www.symplur.com/blog/healthcare-hashtags-socialproject/. 